\title{
DEFINABLE LINEAR ORDERS DEFINABLY EMBED INTO LEXICOGRAPHIC ORDERS IN O-MINIMAL STRUCTURES
}

\author{
JANAK RAMAKRISHNAN \\ (Communicated by Julia Knight)
}

\begin{abstract}
We classify definable linear orders in o-minimal structures expanding groups. For example, let $(P, \prec)$ be a linear order definable in the real field. Then $(P, \prec)$ embeds definably in $\left(\mathbb{R}^{n+1},<_{\text {lex }}\right)$, where $<_{\text {lex }}$ is the lexicographic order and $n$ is the o-minimal dimension of $P$. This improves a result of Onshuus and Steinhorn in the o-minimal group context.
\end{abstract}

\section{INTRODUCTION}

Linear orders are defined by a simple relation, but analyzing them can be quite difficult. In this paper, we consider linear orders definable in densely ordered ominimal groups and give a complete characterization. The study of objects definable in o-minimal structures is an active one HOP10, HO10, since o-minimal structures are "tame" and yet can be expressive enough to define objects of interest to a wide variety of other mathematical areas PS04. Included among o-minimal structures are the field of reals vdD98, and the field of reals with exponentiation and all analytic functions with compact domain vdDM94.

A recent result of Onshuus and Steinhorn implies that any definable linear order in an o-minimal structure $M$ with elimination of imaginaries is a finite union of definable sets, each of which definably embeds in $M^{n}$ for some $n$, ordered lexicographically OS09, Cor. 5.1]. However, this result does not say how elements are compared across sets in the union, and so the ordering is not fully captured by this presentation.

We present an independently discovered characterization of such definable linear orders that completely describes the ordering when the o-minimal structure also defines an order-reversing injection.

Theorem A. Let $M$ be an o-minimal group and let $(P, \prec)$ be an $M$-definable linear order with $n=\operatorname{dim}(P)$. Then there exists an $M$-definable embedding $g$ of $(P, \prec)$ into $M^{2 n+1}$ ordered lexicographically. Moreover, $g(P) \subseteq M^{2 n+1}$ has finite projection to each odd coordinate and $g$ is uniformly definable over the parameters defining $P$.

Received by the editors December 16, 2010 and, in revised form, August 29, 2011.

2010 Mathematics Subject Classification. Primary 03C64; Secondary 06A05.

The author was supported by ANR chaire d'excellence junior THEMODMET (ANR-06-CEXC007). 
Remark 1.1. Although we state the theorem in the group context for simplicity, the proof requires only that $M$ be densely ordered o-minimal, have definable choice, and have a definable order-reversing injection. Moreover, Corollary 1.3 gives a sharper statement when $M$ is a field.

Our characterization improves OS09, Cor. 5.1] in the context of Remark 1.1 since the full order is embedded in a single lexicographic order. This means that the study of definable linear orders in o-minimal structures is just the study of definable subsets of lexicographic orders.

Besides OS09, Cor. 5.1], Theorem A also resembles work done in the general context of embedding ordered sets in lexicographic products [Fle61, CI99]. Seen in that light, Theorem A is a definable version of results in these papers, although the results in the general case are only partial Fle63. Knoblauch Kno00 gives a condition on an order equivalent to its embeddability in a real lexicographic product, but this condition is not easily verifiable for a given class of orders, or even for a specific order.

Outside applications. The study of linear orders has also been undertaken in economics. O-minimality results apply in the economics context because the structure under consideration is usually the reals. When the elements are in a finite Cartesian power, and the functions used to define the linear order are (semi-)algebraicexponential, we are in an o-minimal setting Wil96. Even when the functions are analytic, if the linear order being considered is contained in a bounded set, then we remain in an o-minimal setting vdDMM94.

In BCHIM02a, efforts were made to characterize linear orders that are not order-embeddable in the reals. Theorem A says that any linear order definable in an o-minimal group will be order-embeddable in the reals exactly when it is onedimensional, only finitely many elements have unique predecessors or successors in the order, and the group is itself order-embeddable in the reals.

The desired $g$ in Theorem A can be constructed, given the ability to construct cell decompositions and definable choice functions. We note here that the uniformity in Theorem A follows from a routine model-theoretic compactness argument. Also, it suffices to prove Theorem A for $\emptyset$-definable $P$.

The bound of $2 n+1$ is sharp by the following:

Example 1.2. Let $M=(\mathbb{R},<,+, 0)$ and let $n>0$. Let $P=\left\{\left\langle x_{1}, \ldots, x_{2 n+1}\right\rangle \in\right.$ $M^{2 n+1}: x_{i} \in\{0,1\}$ for $i$ odd $\}$. Let $\prec$ be the lexicographic order on $P$.

There is no embedding of $(P, \prec)$ into a lexicographic order of lower dimension, due to the lack of definable injections between $M$ and proper subsets of $M$. However, given appropriate maps, we have:

Corollary 1.3. If $M$ is an o-minimal field, then in Theorem $A$ the codomain of $g$ can be taken to be $M^{n+1}$, with $g(P)$ having finite projection to the last coordinate.

We will give the proof of Corollary 1.3 after that of Theorem A.

The proof of Theorem A goes by induction. The general case requires techniques to reduce the dimension, whereas the 1-dimensional case is more of a proof by taxonomy. 


\section{NOTATION AND PRELIMINARIES}

We write "definable" to mean " $\emptyset$-definable". We restrict the o-minimal structures we consider to be densely-ordered o-minimal. Throughout, $M$ is an arbitrary fixed o-minimal structure and $P$ a linear order definable in $M$ with $n=\operatorname{dim}(P)$.

Definition 2.1. For $m \geq i \geq 1$, let $\pi_{i}^{m}: M^{m} \rightarrow M$ be projection onto the $i$-th coordinate. Let $\pi_{<i}^{m}: M^{m} \rightarrow M^{i}$ be the map sending $x$ to the $i$-tuple $\left\langle\pi_{1}^{m}(x), \ldots, \pi_{i}^{m}(x)\right\rangle$, and similarly for $\pi_{<i}^{m}$ and $\pi_{>i}^{m}$. We just write $\pi_{i}, \pi_{\leq i}$, etc., since $m$ is always clear from the context. For $x \in M^{m}$, let $x_{i}=\pi_{i}(x)$, and similarly for $x_{\leq i}, x_{<i}$, and $x_{>i}$.

Definition 2.2. Let $<_{\text {lex }}$ denote lexicographic order. A function $g$ is a flexembedding of $P$ if $g$ is an embedding of $(P, \prec)$ into $\left(M^{2 n+1},<_{\operatorname{lex}}\right)$, with $\pi_{i}(g(P))$ a finite set for $i$ odd.

Given first-order structures $M \subseteq N$, we have $M \preceq N$ ( $N$ is an elementary extension of $M)$ if for every formula $\varphi(x)$ with $x$ an $n$-tuple and $a \in M^{n}$, we have $M \models \varphi(a)$ if and only if $N \models \varphi(a)$. A type over a set $A$ is a (possibly) infinite set of formulas in some fixed number of variables, with parameters coming from $A$. Given any number of consistent types over $A \subseteq M$, there is $N$, an elementary extension of $M$, realizing all these types, meaning that for each type, $N$ contains a tuple of elements simultaneously satisfying every formula in the type. See Hod93] for a model theory reference.

O-minimal structures have a number of important properties that will be implicitly used throughout the following. Here, we recall the ones we will be using most often. An excellent reference is vdD98.

O-minimal structures have uniform finiteness: if $\varphi(x, y)$ is a first-order formula with $x$ a singleton and $y$ an $n$-tuple, then there is some natural number $k$ such that for any $M \preceq N$ and $b \in N^{n}$, if $|\varphi(N, b)|>k$, then $\varphi(N, b)$ is an infinite set $\operatorname{vdD98}$, Ch. 3(2.13)].

Cells are a basic building block in o-minimality. If a cell is a subset of $M$, then it is either a point or an interval ("interval" always denotes "open interval"). A cell in $M^{n}$ should be thought of as a slight generalization of a hyperplane in $M^{n}$, of dimension $\leq n$. Each cell in $M^{n}$ has a dimension $\leq n$ vdD98, Ch. 4(1.1)]. The main properties of a cell in $M^{n}$ that we use directly are that for any $k \leq n$, the projection of a cell $C \subseteq M^{n}$ to the first $k$ coordinates is a cell; and if $\operatorname{dim}\left(\pi_{<k}(C)\right)=$ $\operatorname{dim}\left(\pi_{\leq k}(C)\right)$, then $\pi_{\leq k}(C)=\left\{x: x_{<k} \in \pi_{<k}(C) \wedge x_{k}=f\left(x_{<k}\right)\right\}$, for some definable $(k-1)$-ary function $f$ continuous on $\pi_{<k}(C)$ vdD98, Ch. 3(2.3)].

A fundamental fact in o-minimality is cell decomposition of definable sets. That is, given definable sets $A_{1}, \ldots, A_{m} \subseteq M^{n}$, there is a finite partition $\mathcal{C}$ of $M^{n}$ such that each $C \in \mathcal{C}$ is a cell and either disjoint from or contained in $A_{i}$ for $i=1, \ldots, n$. As well, given definable functions $f_{1}, \ldots, f_{m}$ on $A_{1}, \ldots, A_{m}$, we can also suppose that on each $C$, each $f_{i}$, if defined, is continuous and monotonic. Moreover, if $A_{1}, \ldots, A_{m}$ and $f_{1}, \ldots, f_{m}$ are defined using parameters, then the cell decomposition can be taken to vary uniformly in these parameters vdD98. Ch. 3(2.11)].

Fact 2.3. O-minimal structures have "definability of dimension"; that is, given a definable family of sets in $M^{n}$ for some $n$, say $\left\{A_{x} \subseteq M^{n}: x \in B\right\}$, the set $B(k)=$ $\left\{x \in B: \operatorname{dim}\left(A_{x}\right)=k\right\}$ is definable for $k=0, \ldots, n$. Moreover, $\operatorname{dim}(\{\langle x, y\rangle: x \in$ $\left.\left.B(k), y \in A_{x}\right\}\right)=k+\operatorname{dim}(B(k))$ vdD98, Ch. 4(1.5)]. 
Definition 2.4. Let $\mathcal{C}$ be a cell decomposition of $M^{m}$, and let $B \subseteq M^{m}$. Define $\mathcal{C} \cap B=\{C \in \mathcal{C}: C \subseteq B\}$. We say that $\mathcal{C}$ is compatible with $B$ if, for every $C \in \mathcal{C}$, either $C \cap B=\emptyset$ or $C \subseteq B$. We say that $\mathcal{C}$ has good projection if, for any $i<m$ and $C, C^{\prime} \in \mathcal{C}$, either $\pi_{\leq i}(C) \cap \pi_{\leq i}\left(C^{\prime}\right)=\emptyset$ or $\pi_{\leq i}(C)=\pi_{\leq i}\left(C^{\prime}\right)$.

The following is a straightforward application of cell decomposition.

Fact 2.5. Let $\mathcal{C}$ be a definable cell decomposition of $M^{m}$. There is a definable cell decomposition $\mathcal{D}$ that refines $\mathcal{C}$ and has good projection.

\section{1-DIMENSIONAL DEFINABLE LINEAR ORDERS}

For a 1-dimensional cell $C \subseteq M^{m}$, the order $<$ induces an order on $C$ in a natural way via the $p_{C}$ function of $\operatorname{vdD98}$, Ch. 3(2.7)]: for $x, y \in C$, we have $x<y$ if and only if $p_{C}(x)<p_{C}(y)$, since here $p_{C}$ is just the projection bijectively taking $C$ onto the first coordinate on which it has an infinite projection.

A version of the following lemma is folklore, due to C. Steinhorn, with variants stated in [HO10] and OS09]. We need a slightly different statement, and so we prove it here for completeness.

Lemma 3.1. Let $M$ be an o-minimal structure satisfying the conditions of Remark 1.1, and let $(P, \prec)$ be a definable linear order with $\operatorname{dim}(P)=1$. Then $P$ is definably isomorphic to a finite union of disjoint cells on each of which the induced $<$ and the induced $\prec$ agree.

Proof. Let $\mathcal{C}$ be a cell decomposition of $P$. Fix a one-dimensional $C \in \mathcal{C}$, and let $I=p_{C}(C)$. The order $\prec$ induces a linear order on $I$. For $x \in I$, let $G(x)=$ $\{y \in I: x \prec y\}$. Let $\mathcal{J}$ be a cell decomposition of $I$ such that for each $J \in \mathcal{J}$, for all $x \in J$ the set $G(x)$ has the same number of connected components, and the functions defining the boundaries of the infinite connected components of $G(x)$ are monotonic and continuous. Fix an interval $J \in \mathcal{J}$, and let $L=\left\{f_{1}, \ldots, f_{m}\right\}$ and $U=\left\{g_{1}, \ldots, g_{m}\right\}$ be these respectively lower- and upper-boundary-defining functions for $x \in J$. By uniform finiteness of families for o-minimal structures and transitivity of $\prec$, there are only finitely many $x \in I$ with $|G(x)|$ finite, so $m>0$.

Some function in $L \cup U$ must be nonconstant on $J$, since otherwise $G(x)$ and $G(y)$ differ by a bounded finite number of points for $x, y \in J$, which easily violates $\prec$ being a linear order on $J$. We show that the functions in $L \cup U$ "accord", that if some $f \in L$ is increasing, then no $f^{\prime} \in L$ is decreasing and no $g \in U$ is increasing, and similarly for the other possibilities. Assume that we have $f_{i} \in L$ increasing and $f_{j} \in L$ decreasing for some $i, j \leq m$. If $x<y \in J$ with $y$ sufficiently close to $x$, then $f_{j}(y)<f_{j}(x)<g_{j}(y)$. So $\left(f_{j}(y), g_{j}(y)\right) \backslash G(x) \neq \emptyset$ and $\left(f_{i}(x), g_{i}(x)\right) \backslash G(y) \neq \emptyset$, so we have both $G(y) \not \subset G(x)$ and $G(x) \not \subset G(y)$, which is impossible since $\prec$ is a total order. The arguments for $g_{i}, g_{j} \in U$ and for $f_{i} \in L, g_{j} \in U$ are similar.

This "accord" easily implies that $\prec$ is either increasing or decreasing on $J$. Let $\theta$ be a definable order-reversing injection on $M$. Fix distinct definable $a_{J} \in M$ for $J \in \mathcal{J}$, and let $J^{\prime}$ be $\left\{a_{J}\right\} \times J$ if $\prec$ is increasing on $J$, and $\left\{a_{J}\right\} \times \theta(J)$ otherwise. Then $\left\{J^{\prime}: J \in \mathcal{J}\right\}$ is a disjoint collection of cells on each of which the induced $\prec$ and induced $<$ agree. Repeating this procedure for each $C \in \mathcal{C}$, we are done. 
We can now prove Theorem A for 1-dimensional structures 11 We use Lemma 3.1 to break up a definable linear order into cells, on each of which the order and the structure's order agree. We must then analyze how these pieces fit together in the definable order.

Theorem 3.2. Let $M$ be an o-minimal structure satisfying the conditions of Remark 1.1, and let $(P, \prec)$ be a definable linear order with $\operatorname{dim}(P)=1$. Then there exists a definable $g$, a flex-embedding of $P$.

Proof. We say that a lexicographically ordered subset of $M^{3}$ with finite projections to the first and third coordinates is "nice".

By Lemma 3.1. we can suppose that $P$ has a cell decomposition, $\mathcal{D}$, such that $\prec$ is increasing on each $D \in \mathcal{D}$ with respect to the induced $<$. Let $k=|\mathcal{D}|$. We show the theorem by induction on $k$. The case $k=1$ is trivial, since the unique cell $D \in \mathcal{D}$ maps via the $p_{D}$ function into $M$ and then to $\{0\} \times M \times\{0\}$. We prove the case $k$, given it for case $k-1$. Fix $D \in \mathcal{D}$ and replace $P$ by $P^{\prime} \cup I$, where (i) $P^{\prime}$ is a nice subset of $M^{3}$ that is the image of the embedding of $\mathcal{D} \backslash\{D\}$ into $M^{3}$ given by induction, (ii) $I=p_{D}(D)$ is a point or an interval in $M$, and (iii) $\prec$ and $<$ agree on $I$. Our concern is how $I$ and $P^{\prime}$ interact.

Claim 3.3. There is a cell decomposition of $I$ such that for each cell $C$ in the decomposition, one of the following holds:

(PI) For each $x \in C$ there is $y \in P^{\prime}$ with $y$ the immediate $\prec$-successor of $x$ (that is, $x \prec y$ and $\left.(x, y)_{\prec}=\emptyset\right)$.

(PII) For each $x \in C$ there is $y \in P^{\prime}$ with $y$ the immediate $\prec$-predecessor of $x$.

(PIII) Every element of $C$ lies in the same $\prec$-cut in $P^{\prime}$.

Proof. We consider a cell decomposition of $I$ compatible (Definition 2.4) with the subsets defined by the following conditions on a point $x \in I$ and show that this cell decomposition will satisfy the claim after finitely many subdivisions.

\section{Conditions.}

(C1) there exists $y \in P^{\prime}$ the immediate $\prec$-successor of $x$;

(C2) there exists $y \in P^{\prime}$ the immediate $\prec$-predecessor of $x$;

(C3) there exists $y \in P^{\prime}$ with $x \prec y$ and $(x, y)_{\prec} \cap P^{\prime}=\emptyset$ but (C1) fails;

(C4) there exists $y \in P^{\prime}$ with $y \prec x$ and $(y, x)_{\prec} \cap P^{\prime}=\emptyset$ but (C2) fails;

(C5) $x \succ P^{\prime}$ or $x \prec P^{\prime}$.

Claim 3.4. Let $B \subseteq I$ be a definable set such that none of the above conditions holds on $x$, for all $x \in B$. Then $B$ realizes finitely many $\prec$-cuts in $P^{\prime}$.

Proof. Let $h_{1}(x)=\max \left\{\pi_{1}(y): y \in P^{\prime}, y \prec x\right\}$. Since $P^{\prime}$ is nice, $\pi_{1}\left(P^{\prime}\right)$ is a finite set, and so $h_{1}(x)$ takes only finitely many possible values for $x \in B$. Partitioning $B$, we suppose that $h_{1}(x)$ is constant on $B$, given by $h_{1}$. Furthermore, we suppose that for every $x \in B$, there is $y \in P^{\prime}$ with $\pi_{1}(y)=h_{1}$ and $x \prec y$, since the set of $x \in B$ for which such a $y$ does not exist is definable, and all such $x$ lie in the same $\prec$-cut of $P^{\prime}$.

\footnotetext{
${ }^{1}$ After proving the result, we were informed by C. Steinhorn that he already had a version of it earlier.
} 
Let $h_{2}(x)=\sup \left\{\pi_{2}(y): y \in P^{\prime}, y \prec x, \pi_{1}(y)=h_{1}\right\}$. By the "furthermore" supposition, $h_{2}(x) \in M$ for $x \in B$. Assume that for some $a \in B$, there exists $b \in M$ with $\left\langle h_{1}, h_{2}(a), b\right\rangle \in P^{\prime}$. By the niceness of $P^{\prime}$, there are only finitely many $y \in P^{\prime}$ with $\pi_{\leq 2}(y)=\left\langle h_{1}, h_{2}(a)\right\rangle$. But then $a$ must satisfy one of (C1)-(C4) with some such $y$, a contradiction. Thus $P^{\prime}$ contains no elements with the first two coordinates $\left\langle h_{1}, h_{2}(x)\right\rangle$ for any $x \in B$, so in $P^{\prime}$, the $\prec$-cut of $x \in B$ and the $<_{\text {lex }}$-cut of $\left\langle h_{1}, h_{2}(x), 0\right\rangle$ are the same.

We can then show that the elements of $B$ realize finitely many $\prec$-cuts in $P^{\prime}$ by showing that the set $h_{2}(B)$ is finite. If $h_{2}(B)$ were infinite, then it would contain an interval, but this is impossible, since $\left\langle h_{1}, h_{2}(x)\right\rangle \notin \pi_{\leq 2}\left(P^{\prime}\right)$ for any $x \in B$, and $h_{2}$ is defined as a sup of elements in $\pi_{2}\left(P^{\prime}\right)$.

For any $B \subseteq I$, we say that $B$ satisfies one of (C1)-C5 if that condition holds for all $x \in B$. Let $\mathcal{C}$ be a cell decomposition of $I$ compatible with the sets defined by (C1)- (C5).

Claim 3.5. Let $B \subseteq I$ be an interval satisfying (C3) (or (C4)). Then $B$ realizes finitely many $\prec$-cuts in $P^{\prime}$.

Proof. For $x \in B$, let $f(x)$ denote the (necessarily unique) element of $P^{\prime}$ with $(x, f(x))_{\prec} \cap P^{\prime}=\emptyset$. Assume the claim fails. Since $f(x)$ determines the $\prec$-cut of $x$ in $P^{\prime}$, the set $f(B)$ is infinite. By a routine dimension argument on fibers, there are infinitely many $x \in B$ such that the set $f^{-1}(f(x))$ is finite. Choose $a$ with $f^{-1}(f(a))$ finite, and in addition with $a=\max \left(f^{-1}(f(a))\right)$. By (C3), we can choose $z \in(a, f(a))_{\prec}$, and actually we can choose such a $z$ in $B$ since $\prec$ agrees with $<$ on $I$. Then since $(z, f(z))_{\prec} \cap P^{\prime}$ and $(a, f(a))_{\prec} \cap P^{\prime}$ are both empty, we get a contradiction. The argument for (C4) is similar.

This proves Claim 3.3, since if $C \in \mathcal{C}$ satisfies (C5), we can partition $\mathcal{C}$ so that every element lies in the same $\prec$-cut of $P^{\prime}$, and, due to Claims 3.5 and 3.4, we can partition each $C \in \mathcal{C}$ satisfying (C3), (C4), or satisfying no conditions, so that all elements lie in the same $\prec$-cut.

Fix a cell decomposition of $I$ satisfying Claim [3.3, $I_{1}<\cdots<I_{m}$. Note that $\left\{I_{2}, \ldots, I_{m}\right\}$ is a cell decomposition of $I \backslash I_{1}$ satisfying Claim 3.3 with respect to $P^{\prime} \cup I_{1}$, since properties ( $(\mathrm{PI})$ and $(\underline{\mathrm{PII}})$ are trivially preserved, and $I_{1} \prec I \backslash I_{1}$ implies that property (PIII) is too.

We will give a definable embedding $g$ of $P^{\prime} \cup I_{1}$ into $M^{3}$ such that the image is still nice. The decomposition $I_{2}, \ldots, I_{m}$ will satisfy Claim 3.3 with respect to $g\left(P^{\prime} \cup I_{1}\right)$ by the above argument, so we will be done by induction on $m$.

If $I_{1}$ satisfies property (PI) of Claim[3.3, let $f: I_{1} \rightarrow P^{\prime}$ be the definable injection with $f(x)$ the unique $y \in P^{\prime}$ such that $y \succ x$ and $(x, y)_{\prec}$ is empty. Let $f_{3}(x)=$ $\pi_{3}(f(x))$. By niceness of $P^{\prime}$, for any $x \in I_{1}$ the set $R_{x}=\left\{z \prec f(x): \pi_{\leq 2}(f(x))=\right.$ $\left.\pi_{\leq 2}(z)\right\}$ is finite. Thus, by definable choice there is some definable function $h$ with $h(x)<f_{3}(x)$ and $\left\langle\pi_{\leq 2}(f(x)), h(x)\right\rangle \succ R_{x}$. Let $g(x)=\left\langle\pi_{\leq 2}(f(x)), h(x)\right\rangle$ for $x \in I$ and extend $g$ on $P^{\prime}$ by the identity. The function $g$ is a definable embedding of the ordered set $P^{\prime} \cup I_{1}$ into $M^{3}$ ordered lexicographically. For $x \in I_{1}$, we have $\pi_{\leq 2}(f(x))=\pi_{\leq 2}(f(y))$ for only finitely many $y \in I_{1}$, which implies that $g\left(P^{\prime} \cup I_{1}\right)$ is nice.

We proceed analogously if $I_{1}$ satisfies property $(\underline{\mathrm{PI}})$ with respect to $P^{\prime}$. 
Now suppose that $I_{1}$ satisfies property (PIII) with respect to $P^{\prime}$. First suppose that this cut is also satisfied by some element $b \in M^{3}$ with $\pi_{1}(b) \notin \pi_{1}\left(P^{\prime}\right)$, when we consider $P^{\prime}$ embedded as a lexicographic order in $M^{3}$. Then map $I_{1}$ to $\left\langle\pi_{1}(b), I_{1}, 0\right\rangle$, and fix $P^{\prime}$. This map has the desired properties since any two elements with first coordinate $\pi_{1}(b)$ are in the same $\prec$-cut in $P^{\prime}$.

Otherwise, by definable completeness of $(M,<)$ there are $b, b^{\prime} \in P^{\prime}$ with $\pi_{1}(b)=$ $\pi_{1}\left(b^{\prime}\right)$ and $b \prec I_{1} \prec b^{\prime}$. Let $a=\pi_{1}(b)$. Let $B=\left\{x \in P^{\prime}: \pi_{1}(x)=a \wedge x \succ I_{1}\right\}$. Let $c$ be the least element of $\pi_{1}\left(P^{\prime}\right)$ greater than $a$, or $\infty$ if $a=\max \left(\pi_{1}\left(P^{\prime}\right)\right)$. Choose definable elements $d, e \in M$ with $a<d<e<c$. Let $g: P^{\prime} \cup I_{1} \rightarrow M^{3}$ be the identity on $P^{\prime} \backslash B$, and let $g$ send $x \in B$ to $\left\langle e, \pi_{\geq 2}(x)\right\rangle$ and send $I_{1}$ to $\left\langle d, I_{1}, 0\right\rangle$. The map $g$ is a definable embedding of $P^{\prime} \cup I_{1}$ into $M^{3}$. It is easy to see that $g\left(P^{\prime} \cup I_{1}\right)$ is still nice.

\section{4. $n$-DIMENSIONAL DEFINABLE LINEAR ORDERS}

Proof of Theorem $A$. Let $H=\left\{x \in P: \forall y \prec x\left(\operatorname{dim}\left((y, x)_{\prec}\right)=n\right)\right\}$. Points in $H$ have "intrinsically full dimension" below; i.e., any $\prec$-interval approaching one from below has dimension $n$. Note that $H$ is definable.

Lemma 4.1. If $\operatorname{dim}(H)=n$, then $n \leq 1$.

Proof. For each $x \in H$, let $B_{x}=\left\{z \in P:(x, z)_{\prec}\right.$ infinite, $\left.(x, z)_{\prec} \cap H=\emptyset\right\}$. For distinct $x, y \in H$, the sets $B_{x}$ and $B_{y}$ are disjoint, and if $B_{x}$ is nonempty, it has positive dimension. Thus, the set $B=\left\{x \in H: B_{x} \neq \emptyset\right\}$ must have dimension less than $n$.

Let $\Gamma \subseteq H \backslash B$ be a definable connected 1-dimensional set. (To define $\Gamma$, we can take $H^{\prime}$ to be a definable $(n-1)$-dimensional cell in $H \backslash B$, fix definable $\left\langle a_{1}, \ldots, a_{n-1}\right\rangle \in \pi_{<n}\left(H^{\prime}\right)$ and then let $\Gamma$ be the fiber of $H^{\prime}$ over $\left\langle a_{1}, \ldots, a_{n-1}\right\rangle$.) Applying Lemma 3.1 and restricting, we may suppose that $\prec$ agrees with the induced $<$ on $\Gamma$. Let $T: P \rightarrow \Gamma$ be the definable partial function $T(x)=\inf _{\prec \mid \Gamma}\{y \in \Gamma: y \succeq x\}$ when this inf exists. Note that $T(x)$ is defined if there is $z \in \Gamma$ with $z \prec x$. The sets $T^{-1}(y)$ and $T^{-1}(z)$ are $\prec$-convex and disjoint for distinct $y, z \in \Gamma$. By cell decomposition, there is a definable infinite connected set $\Gamma^{\prime} \subseteq \Gamma$ on which $\operatorname{dim}\left(T^{-1}(y)\right)$ is constant. Let $p=\operatorname{dim}\left(T^{-1}(y)\right)$ for $y \in \Gamma^{\prime}$.

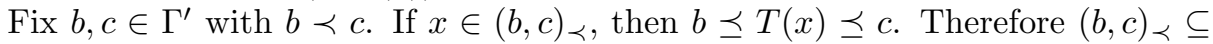
$\bigcup_{y \in \Gamma^{\prime} \cap[b, c]_{\prec}} T^{-1}(y)$, and so $\operatorname{dim}\left((b, c)_{\prec}\right) \leq \operatorname{dim}\left(\bigcup_{y \in \Gamma^{\prime} \cap[b, c]_{\prec}} T^{-1}(y)\right)=1+p \leq n$.

Assume for a contradiction that $p>0$. Fix $a \in \Gamma^{\prime}$. First, assume that there exists $d \in T^{-1}(a)$ with $d \prec a$. Then $(d, a)_{\prec} \subseteq T^{-1}(a)$, so $\operatorname{dim}\left((d, a)_{\prec}\right) \leq p<n$, but since $a \in H$, we have $\operatorname{dim}\left((y, a)_{\prec}\right)=n$ for all $y \prec a$, a contradiction. Thus $T^{-1}(a) \succeq a$. Fix $d \in T^{-1}(a)$ with $(a, d)_{\prec}$ infinite, which is possible since $\operatorname{dim}\left(T^{-1}(a)\right)>0$. Since $a \in H \backslash B$, there is $d^{\prime} \in H \cap(a, d)_{\prec}$, so $\operatorname{dim}\left(\left(a, d^{\prime}\right)_{\prec}\right)=n \leq p<n$, a contradiction.

Thus $p=0$ and $\operatorname{dim}\left((b, c)_{\prec}\right) \leq 1$, so $n \leq 1$.

If Lemma 4.1 holds, then we are done by the 1-dimensional case, so we suppose from now on that $\operatorname{dim}(H)<n$.

Let $E$ be the equivalence relation on $P$ defined as $x E y$ if and only if $\operatorname{dim}\left((x, y)_{\prec} \cup\right.$ $\left.(y, x)_{\prec}\right)<n$. Note that the $E$-classes of $P$ are $\prec$-convex.

Lemma 4.2. No E-class has dimension n. 
Proof. Assume not, so there is an $E$-class $B$ with $\operatorname{dim}(B)=n$. We replace $P$ by $B$. Then for any $x, y \in P, \operatorname{dim}\left((x, y)_{\prec}\right)<n$, but $\operatorname{dim}(P)=n$. Suppose first that $P$ has no endpoints. Consider the partial $M$-types $p_{1}(x)$, which says that $x \in P$ and $x \prec a$ for each $a \in P(M)$; and $p_{2}(x)$, which says that $x \in P$ and $x \succ a$ for each $a \in P(M)$, and let $b_{i} \models p_{i}$ for $i=1,2$ with $b_{1}, b_{2} \in M^{\prime}$, an elementary extension of $M$. Then $\operatorname{dim}\left(\left(b_{1}, b_{2}\right)_{\prec}\right)<n$, since this first-order property of $P$ is preserved in $M^{\prime}$, but $P(M)$ is $n$-dimensional and contained in $\left(b_{1}, b_{2}\right)_{\prec}$, a contradiction. If $P$ has a left endpoint, $c$, modify $p_{1}(x)$ to say $x \prec a$ for each $a \in P(M) \backslash\{c\}$ and $x \succ c$, and similarly if $P$ has a right endpoint.

Lemma 4.2 implies that $E$ has infinitely many equivalence classes. The proof now proceeds through quotienting by $E$.

Claim 4.3. Let $m=\operatorname{dim}(P / E)$. Then $m<n$.

Proof. If not, then there is $B \subseteq P / E$ with $\operatorname{dim}(B)=n$ such that each $E$-class represented in $B$ is finite. Each $E$-class represented in $B$ has a $\prec$-least element. Let $D$ be the set of these $\prec$-least elements, so $\operatorname{dim}(D)=\operatorname{dim}(B)=n$. For any $x \in D$ and any $y \prec x$, we have $\operatorname{dim}\left((y, x)_{\prec}\right)=n$, so if $x \in D$ is not the $\prec$-least element of $P$, then $x \in H$. Thus $\operatorname{dim}(D)=n$ implies $\operatorname{dim}(H)=n$, a contradiction.

The order on $P$ induces a linear order on $P / E$. By induction, there exists $g$, a definable embedding of $(P / E, \prec)$ into $\left(M^{2 m+1},<_{\text {lex }}\right)$, with $g(P / E)$ having finite projection to each odd coordinate. Then $P$ is definably isomorphic as an order to $\left\{\langle x, y\rangle: x \in g(P / E), y \in\left[g^{-1}(x)\right]_{E}\right\}$, ordered lexicographically by the orders $<_{\text {lex }}$ on $g(P / E)$ and $\prec$ on $\left[g^{-1}(x)\right]_{E}$ for $x \in P / E$. We replace $P$ by this ordered set and $P / E$ by $g(P / E)$. Let $Q_{x}=[x]_{E}$, ordered by $\prec$, for $x \in P / E$. The remainder of the proof is just to bound the dimension of the embedding of $P$, since it is easy to embed the orders $Q_{x}$ uniformly in some lexicographic order.

Compressing $P / E$. Let $\mathcal{C}$ be a cell decomposition of $P / E$ with good projection (Definition 2.4) such that, for $C \in \mathcal{C}$, if $x, y \in C$, then $\operatorname{dim}\left(Q_{x}\right)=\operatorname{dim}\left(Q_{y}\right)$.

We must "compress" each $C \in \mathcal{C}$ while preserving the lexicographic order. For $i \leq 2 m+1$ and $C \in \mathcal{C}$, let

$$
V_{i}(C)=\left\{D \in \pi_{\leq i}(\mathcal{C}): \pi_{<i}(D)=\pi_{<i}(C), D \neq \pi_{\leq i}(C)\right\},
$$

the set of projections in $\mathcal{C}$ that agree with $C$ up to the $(i-1)$-st coordinate but disagree at the $i$-th coordinate. It is these cells that may pose problems if we modify the $i$-th coordinate of $C$. Let $k(j, C)$ be the greatest coordinate $k$ such that $\operatorname{dim}\left(\pi_{\leq k}(C)\right)=\operatorname{dim}\left(\pi_{\leq j}(C)\right)$, and let $V(j, C)=\bigcup_{j<i \leq k(j, C)} V_{i}(C)$. The collection $V(j, C)$ represents cells that must be shifted before the coordinates of $C$ can be collapsed. If $V(j, C)=\emptyset$, then transforming $C$ by modifying coordinates $>j$ will not affect the ordering between elements of $C$ and the rest of $P / E$.

Fix odd $j$ minimal and $C \in \mathcal{C}$ such that $V(j, C) \neq \emptyset$, and let $k=k(j, C)$. For each $i \in(j, k]$, let $r(i)=\left|V_{i}(C)\right|$. Each $V_{i}(C) \cup\left\{\pi_{\leq i}(C)\right\}$ is totally ordered by the relation $D<_{i} D^{\prime}$ given by

$$
\forall x \in \pi_{<i}(C)\left(\{y:\langle x, y\rangle \in D\}<\left\{y:\langle x, y\rangle \in D^{\prime}\right\}\right) .
$$

Let $s(i)=\left|\left\{D \in V_{i}(C): D<_{i} C\right\}\right|$. Let $\pi_{j}(C)=\{b\}$. For $i=j+1, \ldots, k$, fix definable $c_{1}^{i}<\cdots<c_{r(i)}^{i}$ in $M$ such that the following three properties hold: 
$c_{s(i)}^{i}<b<c_{s(i)+1}^{i}$; for $i>j+1$ we have $c_{s(i-1)}^{i-1}<c_{1}^{i}<c_{r(i)}^{i}<c_{s(i-1)+1}^{i-1}$; and $\left(c_{1}^{j+1}, c_{r(i)}^{j+1}\right) \cap \pi_{j}(P / E)=\{b\}$. The elements $c^{i}$ lie in nested intervals around $b$. Then let $h: P / E \rightarrow M^{2 m+1}$ be defined by $h(x)=x$ if $\pi_{\leq i}(x) \notin \bigcup V(j, C)$ for all $i>j$, and otherwise, $h(x)=\left\langle x_{<j}, c_{t}^{i}, x_{>j}\right\rangle$, where $\pi_{\leq i}(\bar{x})$ belongs to the $t$-th member of the $<_{i}$-ordered set $V_{i}(C)$. The function $h$ maps the finitely many ways in which a cell can "branch" from $C$, at coordinates $j+1, \ldots, k$, into the $j$-th coordinate.

The function $h$ is an embedding of $\left(P / E,<_{\text {lex }}\right)$ and the set $h(\mathcal{C})$ is a cell decomposition of $h(P / E)$ with good projection. Moreover, if we let $V^{h}$ be defined as $V$ was but for $h(P / E)$, then for any $C^{\prime} \in h(\mathcal{C})$ and any odd $j^{\prime}<2 m$, we have $\left|V^{h}\left(j^{\prime}, h\left(C^{\prime}\right)\right)\right| \leq\left|V\left(j^{\prime}, C^{\prime}\right)\right|$, and in particular $V^{h}(j, h(C))=\emptyset$. Thus, after replacing $P / E$ by $h(P / E)$ and repeating this finitely many times, we may suppose that $V(j, C)=\emptyset$ for all odd $j$ and all $C \in \mathcal{C}$.

Fix $C \in \mathcal{C}$ and let $k(C)$ be the first odd coordinate $k$ such that $\operatorname{dim}\left(\pi_{\leq k+1}(C)\right)=$ $\operatorname{dim}\left(\pi_{\leq k}(C)\right)$, or $2 m+1$ otherwise. Let $O=\left\{j \in(k(C), 2 m]: \operatorname{dim}\left(\pi_{\leq j}(C)\right)>\right.$ $\operatorname{dim}\left(\pi_{<j}(C)\right\}$, and let $O=\{j(1), \ldots, j(r)\}$. Define $h_{C}: C \rightarrow M^{2 m+1}$ by $h_{C}(x)=$ $\left\langle x_{\leq k(C)}, x_{j(1)}, 0, x_{j(2)}, 0, \ldots, x_{j(r)}, 0, \ldots, 0\right\rangle$, and let $h$ be the union of the functions $h_{C}$. We show that $h$ is an order-preserving embedding of $P / E$. For distinct $C_{1}, C_{2} \in$ $\mathcal{C}$, let $s=\min \left(k\left(C_{1}\right), k\left(C_{2}\right)\right)$. Since $V\left(k\left(C_{i}\right), C_{i}\right)=\emptyset$ for $i=1,2$, if $x \in C_{1}$ and $y \in C_{2}$, then $\pi_{\leq s}(x) \neq \pi_{\leq s}(y)$, and since $\pi_{\leq s}(h(x))=\pi_{\leq s}(x)$, and similarly for $y$, the map $h$ must preserve the ordering on $x$ and $y$. Thus, we can restrict to a single $C$. Let $x, y \in C$. Let $i$ be the first coordinate such that $x_{i} \neq y_{i}$. If $i<k(C)$, then $\pi_{\leq i}(h(x))=\pi_{\leq i}(x)$ and similarly for $y$, and we are done. Thus $i \in O$, so $i=j(t)$ for some $t \leq r$. By definition of $h$, the first coordinate at which $h(x)$ and $h(y)$ differ is $l=k(C)+2 t-1$, at which $\pi_{l}(h(x))=\pi_{i}(x)$ and $\pi_{l}(h(y))=\pi_{i}(y)$, so we have shown that $h$ is an order-preserving embedding of $P / E$. Moreover, $h(\mathcal{C})$ is a cell decomposition of $h(P / E)$.

After replacing $P / E$ by $h(P / E)$ and $\mathcal{C}$ by $h(\mathcal{C})$, we have $\pi_{i}(C)=\{0\}$ for all $i>2 \operatorname{dim}(C)+1$ and $C \in \mathcal{C}$.

Compressing $Q_{x}$. For $C \in \mathcal{C}$, let $q(C)=\operatorname{dim}\left(Q_{x}\right)$ for $x \in C$. By Lemma 4.2, $q(C)<n$. By induction for Theorem A, each $Q_{x}$ can be definably flex-embedded in $M^{2 q(C)+1}$, and since $Q_{x}$ is uniformly definable in $x$, this embedding can be taken to be uniform as well, so we have a definable function $g_{C}$ with $g_{C}(x,-)$ a flexembedding of $Q_{x}$ for all $x \in C$. Letting $q=\max \{q(C): C \in \mathcal{C}\}$, we can embed each $M^{2 q(C)+1}$ in $M^{2 q+1}$, extending by 0 , and so suppose that each $g_{C}$ embeds into $M^{2 q+1}$, and let $g$ be their union. Replace $P$ by $\left\{\langle x, g(x, y)\rangle: x \in P / E, y \in Q_{x}\right\}$, so $Q_{x}$ is replaced by $g\left(x, Q_{x}\right)$. For each odd $i \leq 2 q+1$ and $x \in P / E$, the set $\pi_{i}\left(Q_{x}\right)$ is finite. Thus, by o-minimality $\left|\pi_{i}\left(Q_{x}\right)\right|$ is bounded as $x$ ranges over $P / E$, and so we can set $r=\max \left\{\left|\pi_{i}\left(Q_{x}\right)\right|\right.$ : odd $\left.i \leq 2 q+1, x \in P / E\right\}$ and fix definable $a_{1}<\cdots<a_{r} \in M$. Then define $h_{x}: Q_{x} \rightarrow M^{2 q+1}$ so that $\pi_{i}\left(h_{x}(y)\right)=y_{i}$ for $i$ even and $\pi_{i}\left(h_{x}(y)\right)=a_{t}$ for $i$ odd, with $y_{i}$ the $t$-th element in the finite ordered set $\pi_{i}\left(Q_{x}\right)$. Replace $P$ by $\left\{\left\langle x, h_{x}(y)\right\rangle: x \in P / E, y \in Q_{x}\right\}$ and $Q_{x}$ by $h_{x}\left(Q_{x}\right)$.

Joining. We now have $P \subseteq M^{2(m+q)+2}$, ordered lexicographically, with $\pi_{i}(P)$ finite for odd $i \leq 2 m+1$ and even $i \geq 2 m+2$. Let $m(C)=\operatorname{dim}(C)$ for $C \in \mathcal{C}$. For each $C \in \mathcal{C}$, we have $\pi_{i}(C)=\{0\}$ if $2 m(C)+1<i \leq 2 m+1$, and for $x$ with $\pi_{\leq 2 m+1}(x) \in C$, we have $\pi_{i}(x)=\{0\}$ if $2 m+1+2 q(C)+1<i \leq 2(m+q)+2$. 
For each $C \in \mathcal{C}$, let $\pi_{2 m(C)+1}(C)=\left\{b^{C}\right\}$. Fix definable $c_{1}^{C}<\cdots<c_{r}^{C}$ such that $b^{C} \in\left(c_{1}^{C}, c_{r}^{C}\right)$ and $\left(c_{1}^{C}, c_{r}^{C}\right) \cap\left(c_{1}^{D}, c_{r}^{D}\right)=\emptyset$ for $D \neq C$. Let $g_{C}$ take $x \in P$ with $\pi_{\leq 2 m+1}(x) \in C$ to $\left\langle x_{\leq 2 m(C)}, c_{t}, x_{2 m+3}, x_{2 m+4}, \ldots, x_{2 m+2 q(C)+2}\right\rangle$, where $x_{2 m+2}$ is the $t$-th element of the ordered set $\left\{y:\left\langle x_{<2 m+2}, y\right\rangle \in \pi_{\leq 2 m+2}(P)\right\}$. Note that this ordered set has at most $r$ elements. The codomain of $g_{C}$ is $M^{2 m(C)+2 q(C)+1}$. Since $m(C)+q(C) \leq n$, we can take all the functions $g_{C}$ to map to $M^{2 n+1}$ through extending by 0 . Then the union of the functions $g_{C}$ is the desired embedding.

Proof of Corollary 1.3. The bound in Corollary 1.3 comes from taking the image of $g(P)$ under embeddings whose existence is guaranteed by the following:

Claim 4.4. Let $M$ be an o-minimal field. Let $B \subset M^{n}$ be definable, with $\left|\pi_{k}(B)\right|$ finite for some $k<n$. Then $\left(B,<_{\text {lex }}\right)$ embeds definably into $\left(M^{n-1},<_{\text {lex }}\right)$.

Proof. Let $\pi_{k}(B)=\left\{a_{1}<\cdots<a_{m}\right\}$. Let $a_{0}=-\infty$. For $i \leq m$, let $f_{i}$ : $M \rightarrow\left(a_{i-1}, a_{i}\right)$ be a definable order-preserving injection. For $x \in B$, let $h(x)=$ $\left\langle x_{<k}, f_{i}\left(x_{k+1}\right), x_{>k+1}\right\rangle$, when $x_{k}=a_{i}$. Then $h$ is the desired embedding.

\section{ACKNOWLEDGements}

The author would like to thank C. Steinhorn for an informative discussion when he first learned of Steinhorn's results with A. Onshuus, and a later discussion that helped clarify the direction of this paper, as well as F. Wagner for an encouraging talk on generalizing the result in the absence of a field. The author also thanks the anonymous referee for careful comments and suggestions.

\section{References}

[BCHIM02a] Alan F. Beardon, Juan C. Candeal, Gerhard Herden, Esteban Induráin, and Ghanshyam B. Mehta. The non-existence of a utility function and the structure of non-representable preference relations. J. Math. Econom., 37(1):17-38, 2002. MR.1901054 (2003e:91063)

[CI99] Juan C. Candeal and Esteban Induráin. Lexicographic behaviour of chains. Arch. Math. (Basel), 72(2):145-152, 1999. MR1667055 (2000a:06001)

[Fle61] I. Fleischer. Embedding linearly ordered sets in real lexicographic products. Fund. Math., 49:147-150, 1960/1961. MR0124243 (23:A1557)

[Fle63] Isidore Fleischer. Correction to "Embedding linearly ordered sets in real lexicographic products". Norske Vid. Selsk. Forh. (Trondheim), 36:34-35, 1963. MR0150055 (27:58)

[HO10] Assaf Hasson and Alf Onshuus. Unstable structures definable in o-minimal theories. Selecta Math. (N.S.), 16(1):121-143, April 2010. MR2609645

[Hod93] Wilfrid Hodges. Model Theory. Cambridge University Press, 1993. MR1221741 (94e:03002)

[HOP10] Assaf Hasson, Alf Onshuus, and Ya'acov Peterzil. Definable structures in o-minimal theories: one dimensional types. Israel J. Math., 179:363-379, 2010. MR2735047

[Kno00] Vicki Knoblauch. Lexicographic orders and preference representation. J. Math. Econom., 34(2):255-267, 2000. MR1776406(2001d:91058)

[OS09] Alf Onshuus and Charles Steinhorn. On linearly ordered structures of finite rank. J. Math. Log., 9(2):201-239, 2009. MR2679440

[PS04] Ya'acov Peterzil and Sergei Starchenko. Uniform definability of the Weierstrass $\wp$ functions and generalized tori of dimension one. Selecta Math. (N.S.), 10(4):525-550, 2004. MR2134454 (2006d:03063) 
[vdD98] Lou van den Dries. Tame topology and o-minimal structures, volume 248 of London Mathematical Society Lecture Note Series. Cambridge University Press, Cambridge, 1998. MR 1633348(99j:03001)

[vdDM94] Lou van den Dries and Chris Miller. On the real exponential field with restricted analytic functions. Israel J. Math., 85(1-3):19-56, 1994. MR1264338 (95e:03099)

[vdDMM94] Lou van den Dries, Angus Macintyre, and David Marker. The elementary theory of restricted analytic fields with exponentiation. Ann. of Math. (2), 140(1):183-205, 1994. MR 1289495 (95k:12015)

[Wil96] A. J. Wilkie. Model completeness results for expansions of the ordered field of real numbers by restricted Pfaffian functions and the exponential function. J. Amer. Math. Soc., 9(4):1051-1094, 1996. MR1398816 (98j:03052)

CMaf, University of Lisbon, Av. Prof. Gama Pinto, 2, 1649-003 Lisboa, Portugal

E-mail address: janak@janak.org

URL: http://janak.org/ 